\title{
Chironomidés (Diptera) des Alpes françaises et des basses régions avoisinantes
}

\author{
B. Serra-Tosio 1
}

Mots clés: Diptera, Chironomidae, faunistique, Alpes françaises, montagnes, plaines.

Le catalogue des Chironomidés des Alpes françaises et des basses régions avoisinantes, c'est-à-dire de la portion du territoire français située au sud et à l'est du Rhône (Camargue comprise), est établi à partir des travaux antérieurs. L'aire couverte se présente comme une interpénétration complexe d'une partie de la zone 4 (Alpes au-dessus de $1000 \mathrm{~m}$ d'altitude) et d'une partie de la zone 13 (basses régions occidentales au-dessous de $1000 \mathrm{~m}$ d'altitude) de la Limnofauna Europaea d'Illies (1978).

On a recensé au total 335 espèces, dont 13 nouvelles pour la zone 4 et 123 nouvelles pour la zone 13. En France, le total des espèces de Chironomidés connues des Pyrénées, du Massif Central, des Alpes et des basses régions situées approximativement au sud de la Loire et du Rhône s'élève actuellement à 496.

Chironomids (Diptera) of the French Alps and neighbouring lowland regions.

Keywords : Diptera, Chironomidae, faunistic, French Alps, mountain, lowland.

A catalogue of chironomids from the French Alps and neighbouring lowland regions, i.e. from the part of the French territory situated south and east of the Rhône (including the Camargue) was produced by using previous works. The covered area is a complex interpenetration of a part of area 4 (Alps over $1000 \mathrm{~m}$ ), and a part of area 13 (western lowlands under $1000 \mathrm{~m}$ ) in the Limnofauna Europaea (Illies 1978).

335 species were recorded, with 13 being new records for area 4, and 123 for area 13. For France, the total number of Chironomid species recorded from the Pyrenees, the Massif Central, the Alps and the lowlands situated south of the Loire and the Rhône has now increased to 496.

Afin de faire le point sur l'état de nos connaissances concernant les Diptères Chironomidés de France, une série d'inventaires ont été publiés depuis quelques années. Ils se rapportent à un certain nombre d'aires géographiques dans lesquelles ces insectes ont fait l'objet d'études assez nombreu. ses. Ces aires ont été tant bien que mal rapportées aux subdivisions biogéographiques qui ont servi à découper la région paléarctique occidentale dans la Limnofauna Europaea de J. Illies (éditions de 1967 et de 1978). Ce sont : les Pyrénées françaises (Laville 1980, Laville \& Vinçon 1986) et le Massif Central (Laville \& Serra-Tosio 1987) où s'interpénètrent les

1. Laboratoire de Zoologie et Hydrobiologie, Université Joseph Fourier (Grenoble 1), B.P. 53 X, 38041 Grenoble Cédex, France. zones 2 et 13 de la Limnofauna pour la première aire, les zones 8 et 13 pour la seconde. La distinction entre les zones 2 et 13 d'une part, 8 et 13 d'autre part repose respectivement sur les altitudes $1000 \mathrm{~m}$ et $500 \mathrm{~m}$. Il est inutile d'insister sur le côté arbitraire d'une telle séparation.

Il faut ajouter à cela un inventaire plus ancien sur le bassin du Doubs dans le Jura français (Verneaux \& Vergon 1974), qui intéresse donc une autre partie de la zone 8 de la Limnofauna.

Or pour les Alpes françaises, il n'existait jusqu'à présent aucune étude similaire.

Je présente donc ici (Tableau I) un inventaire des Diptères Chironomidés collectés dans une portion du territoire français limitée à l'est par la frontière avec la Suisse et l'Italic, au nord et à l'ouest par le 
lac Léman et le Rhône, et au sud par la Méditerranée. Ainsi définie, cette aire comprend non seulement les parties plus ou moins montagneuses de la Savoie, du Dauphiné et de la Provence, mais aussi les vallées, le lit toral provençal, la Crau et, entre le Grand Rhône et le Petit Rhône, la Camargue. Il s'agit donc d'un ensemble très hétérogène qui ressortit à deux zones de la Limnofauna: la zone 4 au-dessus de $1000 \mathrm{~m}$ d'altitude, la zone 13 au-dessous. Ces zones s'interpénètrent là de façon complexe, ce qui rend l'étude biogéographique de cette région très délicate. Comme dans le cas des Pyrénées, il est bien évident que la limite de $1000 \mathrm{~m}$ pour séparer les deux zones est tout à fait arbitraire.

Dans les Alpes françaises et les basses régions avoisinantes ainsi définies, les travaux sur les Chironomidés peuvent être historiquement regroupés en plusieurs ensembles.

Le premier rassemble des publications assez anciennes, qui couvrent approximativement les cinq premières décennies du vingtième siècle. Elles concernent partiellement ou en totalité des Chironomidés collectés dans les Alpes et en Provence par des chercheurs travaillant à l'université de Grenoble (Hesse, Léger, Dorier, Motas, Vaillant) ou à celle de Marseille (Timon-David), mais identifiés par des spécialistes extérieurs, français (Kieffer) ou étrangers (Goetghebuer, Thienemann, Wülker).

Le deuxième ensemble comprend les travaux exécutés depuis les années 60 . A partir de cette date, Laville et Tourenq à Toulouse, ainsi que Serra-Tosio à Grenoble, se spécialisent dans l'étude systématique et écologique des Chironomidés de France. Les premiers identifient le matériel de Camargue, de la Crau et du sud des Alpes, collecté par eux ou par divers autres chercheurs (Moubayed, Nino, Séguier, Champeaux, etc...); le second fait de même dans le nord des Alpes et détermine notamment les speciments récoltés par des chercheurs de troisième cycle en vue de travaux menés à l'université de Grenoble (Barboyon, Buisson, Flueler, Gachet, Gay, Madariaga-Meza, Salman, Wasson).

Pour la présente synthèse, près de cent travaux parus depuis le début du siècle jusqu'à aujourd'hui ont été analysés. La liste exhaustive, sauf erreur ou ornissions, de tous les chironomidés signalés dans la zone ci-dessus est présentée dans le tableau I.

\section{Commentaires sur le Tableau I}

Au total, ce sont 335 espèces de Chironomidés qui ont été signalées des Alpes françaises et des basses régions avoisinantes, depuis la frontière jusqu'au Rhône et à la Méditerranée (Camargue comprise). Elles se décomposent en 35 Tanypodinae, 29 Diamesinae, 4 Prodiamesinae, 143 Orthocladiinae, 70 Chironominae Chironomini, 53 Chironominae Tanytarsini et 1 Telmatogetoninae. On n'y trouve donc pour l'instant aucun représentant des Podonominae ni des Buchonomyinae, alors que ces deux sousfamilles sont connues ailleurs en France (de la Corse pour la première, du sud du Massif Central pour la deuxième).

Mais si on ne considère que les Chironomidés capturés à plus de $1000 \mathrm{~m}$ d'altitude, c'est-à-dire dans la partie française de la zone 4 au sens strict, on ne trouve plus que 5 Tanypodinae, 24 Diamesinae, 1 Prodiamesinae, 62 Orthocladinae, 1 Chironominae Chironomini, 8 Chironominae Tanytarsini et aucun Telmatogetoninae, soit un total de 101 espèces. Le faible nombre de Tanypodinae et de Tany. tarsini recensés et surtout l'absence presque complète des Chironomini prouvent, puisque ces insectes sont beaucoup plus nombreux ailleurs dans les Alpes et dans d'autres massifs montagneux européens, que les recherches sur les Chironomidés des Alpes françaises sont particulièrement insuffisantes.

Le nombre d'espèces nouvelles pour la zone 4 de la Limnofauna Europaea (1978), précédées d'un astérisque $\left(^{*}\right)$ dans le tableau I, s'élève au total à 13 et se décompose en 1 Tanypodinae, 2 Diamesinae et 10 Orthocladiinae.

Le nombre d'espèces nouvelles pour la zone 13 de la Limnofauna Europaea (1978), précédées d'un rond ( ${ }^{\circ}$ dans le Tableau I, s'élève au total à 123, dont 10 Tanypodinae, 16 Diamesinae, 3 Prodiamesinae, 62 Orthocladiinae, 12 Chironomini et 20 Tanytarsini. Ces chiffres peuvent paraître relativement élevés. Ils traduisent en fait à la fois le côté arbitraire de la limite inférieure de la zone 4 (l'altitude $1000 \mathrm{~m}$ ) et l'interpénétration complexes des zones 4 et 13 dans cet te région.

Il aurait èté intéressant de comparer la faune chironomidienne des Alpes françaises avec celle des Pyrénées d'une part, et avec celle du Massif Central d'autre part, sur la base des travaux antérieurs (Laville \& Vinçon 1986 ; Laville \& Serra-Tosio 1987). 
Tableau 1. Inventaire des Chirononidés des Alpes et des basses régions avoisinantes.

Les nombres entre parenthèses renvoient aux références bibliographiques:

(a) : stations situées au-dessus de 1000 d'altitude (zone 4 de la Limnofauna Europaea).

$(\overline{0})$ : stations situées au-dessous de $1000 \mathrm{~m}$ d'altitude (zone 13 de la Limnofauna Europaea).

$(\overline{0})$ : stations situées au-dessus et au-dessous de $1000 \mathrm{~m}$ d'altitude (zones 4 et 13 ).

(0) : stations à altitude non précisée.

* : espèce nouvelle pour la zone 4 de la Limnofauna Europaea (1978).

- : espèce nouvelle pour la zone 13 de la Limnofaura Europaea (1978).

$P$ : espèce déjà connue des Pyrénées, au-dessus $(\underline{P})$ ou au-dessous $(\bar{P})$ de $1000 \mathrm{~m}$ d'altitude.

$M C$ : espèce déjà connue du Massif Central, au-dessus (MC) ou au-dessous $(\overline{M C})$ de $500 \mathrm{n}$.

Les nombres en petits caractères renvoient aux notes qui suivent le tableau I. Dans ces notes, les symonymies indiquées sont uniquenent celles qui concernent les noms d'espèce utilisés dans l'aire étudiée. De plus, les identifications, qui, considérées conme erronées ou imprécises, ont fait l'objet de rectifications dans des publications ultérieures, sont indiquées par "sub" suivi du nom employé dans la publication antérieure.

Les noms de genre placés entre guillemets (") sont douteux, ou provisoirement maintenus dans l'attente d'une meilleure connaissance taxonomique des espèces concernées.

Le point d'interrogation (?) placé avant le numéro d'une citation indique que l'auteur cité n'est pas sûr de son identification.

\section{Tarypodinae}

Ablabesmia longristyla Fitt.: $(\overline{2})(\overline{15})(\overline{33})(\overline{41})(\overline{49})(? \overline{77})(\overline{87}) \quad \underline{P} \underline{\mathbf{M C}}$

A. monilis (L.) : $(\overline{3})(\overline{41})(\overline{48})(\overline{49})(\underline{71})(\overline{82})(\overline{90})$ P $\overline{\mathrm{MC}}$

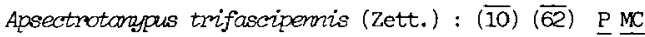

- Arctopelopia barbitarsis (Zett.) : ( $\overline{48}$ )

- A. griseipenis (v.d.W.) : (14) $(\overline{48}) \overline{M C}$

Cinotomprus nemosus (Mg.) : $(\overline{62})(\overline{88})(\overline{90})$

Conchapelopia melanops (Wied.) : $(\overline{\mathrm{IO}})(\overline{15}) \overline{\mathrm{P}} \overline{\mathrm{MC}}$

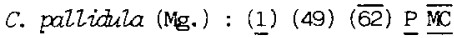

* C. viator (K.) : (1) $(\overline{15})(\overline{49}) \overline{\mathrm{MC}}$

Krenopelopia binotata (Wied.) : $\overline{(\overline{91}}$ )

K. nigropuonctata (Staeg.) : (14)

Macropelopia nebulosa (Mg.) : (프 $(\overline{10})(\overline{15})(\overline{20})(\overline{47})(\overline{69}) \quad \underline{P} \underline{\overline{\mathrm{MC}}}$

M. notata (Mg.) : $(\overline{90}) \underline{\mathrm{P}}$

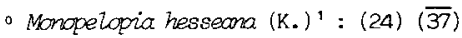

Natarsia pronctata (Fabr.) : (14) $\underline{\mathrm{P}} \overline{\mathrm{MC}}$

- Nitotomapus cabius (Mg.) : $(\overline{18})$ P $\overline{\mathrm{MC}}$ 
Examexina cingulata (Walk.) $)^{2}:$ (14) $(\overline{37})$

Procladius choreus (Mg.) : $(\overline{2})(\overline{15})(? \overline{20})(\overline{33})(\overline{48})(\overline{49})(\overline{62})(\overline{82})(\overline{90})$ P $\overline{\mathrm{MC}}$

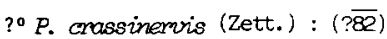

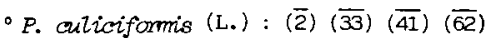

P. parrulus K. : (?क्69) $\overline{\mathrm{P}}$

P. sagittalis $\mathrm{K}$. (?त)

Esectrotonpas vamius (Fabr.) : $(\overline{10})(\overline{41})(\overline{48})(\overline{49})(\overline{77})(\overline{82})(\overline{87})(\overline{90}) \quad \overline{\mathrm{P}} \overline{\mathrm{MC}}$

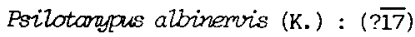

Fheopetopia crnata (Mg.) : $(\overline{14})(\overline{48})(\overline{88})(\overline{90}) \quad \mathrm{P} \overline{\mathrm{MC}}$

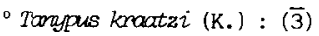

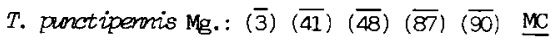

-T. viliperoiis (K.) : $(\overline{82})$

- Thienemonimyia laeta (Mg.) : (14) P

Xenopelopia falcigera (K.) : $(\overline{48})(\overline{82})$

X. nigriocons Fitt.: $(\overline{41})(\overline{48})(\overline{82})(\overline{87})(\overline{90})$

- Zavrelimia barbatipes (K.) : (15)

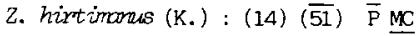

2. melanara (Mg.) : (1) (14) $\underline{P}$

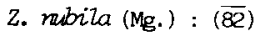

\section{Diamesinae}

* Borecheptagyia alpioola Ser.-Tos.: (81)

* B. dasyops Ser.-Tos.: (81)

B. leger (G.) ${ }^{3}$ : (12) (23) (61) (81) $\mathrm{P} \overline{\mathrm{MC}}$

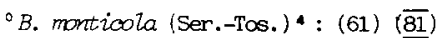

B. rugasa (Saund.) : ( $\overline{61})(\overline{81})$

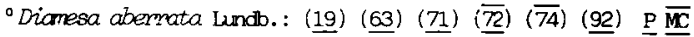

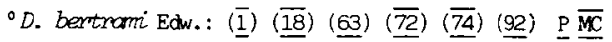

${ }^{\circ} D$. cinerella Mg. : $(\underline{1})(\underline{18})(\underline{19})(\underline{63})(\underline{71})(\overline{72})(\overline{74})(\overline{77})$ P $\underline{\overline{M C}}$

D. dampfi (K.) : (19) (70) (72) (74) (92)

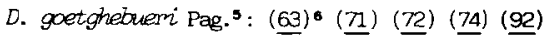

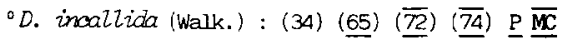

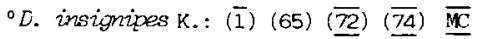


D. Laticardia Ser.-Tos.: (63) (66) (72) (74) (92) $\underline{P}$

o D. Latitamis G.: (1) $(\overline{13})(\overline{17})(\overline{49})(\overline{66})(\overline{72})(\overline{74})(\overline{83})(\overline{85})^{7}(? \overline{91})^{7}$ P

D. iindrothi G.: (65) (72) (74) (92)

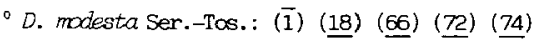

- D. permacra (Walk.) : $(\overline{72})(\overline{74})(791)^{\circ} \mathrm{MC}$

$?^{\circ}$ D. steinboecki G.: $(? \underline{8})^{9}(? \overline{13})^{9}(\underline{19})(\underline{65})(\underline{72})(\underline{74})(\underline{75})(? \overline{85})^{9}(\overline{91})^{9}$ (92) $\underline{\mathrm{P}}$

${ }^{\circ}$ D. tonsa (Hal. $)^{10}:(\overline{1})(\overline{17})(\overline{18})(\underline{19})(\overline{49})(\overline{63})(\underline{71})(\overline{72})(\overline{74})(\overline{77})(\overline{83})$ P $\overline{\mathrm{MC}}$

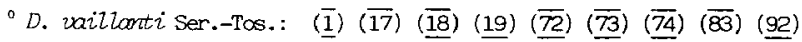

D. whelkeri Ser.-Tos.: (63) (65) (66) (72) (74) $\underline{\mathrm{P}}$

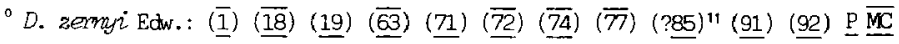

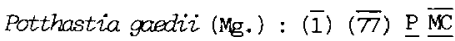

P. Zonginaras K.: (?85) ${ }^{11} \underline{\mathrm{P}} \underline{\overline{\mathrm{MC}}}$

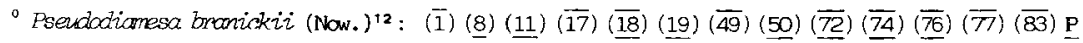

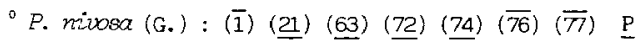

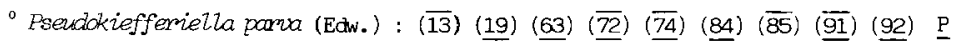
SImpotthastia zavreli Pag.: $(\overline{68})(\overline{72})(\overline{74}) \overline{\mathrm{P}} \overline{\mathrm{MC}}$

- Symitionesa eajurdsi (Pag.) : $(\underline{65})^{13}(\overline{68})(\overline{72})(\overline{74})$ P

\section{Prodiamesinae}

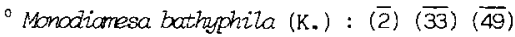

- M. etromi Br.: (49) $\underline{P}$

- Prodiamesa detphinensis Ser.-Tos.: $(\overline{20})(\overline{64})(\overline{7})$

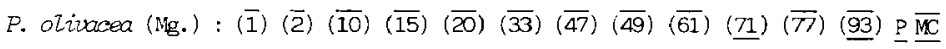

\section{Orthocladiinae}

Acricotopus Zucens (Zet.. $)^{14}:(\overline{48})(\overline{62})(\overline{88})(\overline{90})$

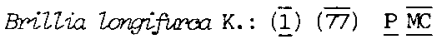

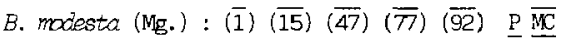

Comptocladius stercorronius (d.Geer) : $(\overline{31})(\overrightarrow{41})(\overrightarrow{49})(\overline{90}) \quad \overrightarrow{\mathrm{P}} \underline{\mathrm{kC}}$

Cortiocladius copuciras (Zett.) : $(\overline{48})(\overline{88})(\overline{90})$ (92) $\underline{P} \overline{\mathrm{MC}}$

- Chaetocladius acminatus Br.: ( $\overline{49})$

Ch. dissipatus (Edw.) : (1)

- Ch. gracilis Br.: (49) $\underline{P}$ 
- Ch. Zaminatus Br.: (1) (91) $\underline{\mathrm{P}}$

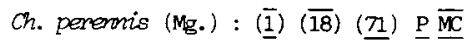

- Commonera bifunata K.: ( $\overline{38})$

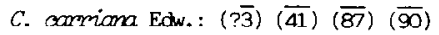

C. cetemipes wim.: ( $\overline{82})$

- C. coronata Edw.: (1)

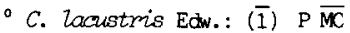

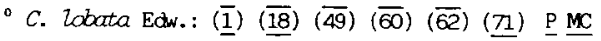

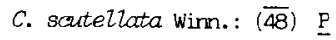

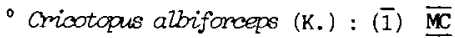

?० C. algorum (K.) : (?T7) $\overline{\mathrm{MC}}$

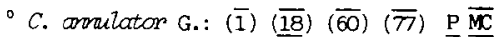

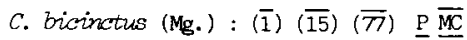

"C. conacellas (K.) : (37)

C. flawocinctus (K.) : $(\overline{49})(\overline{62})(\overline{90}) \overline{\mathrm{P}} \underline{\overline{\mathrm{MC}}}$

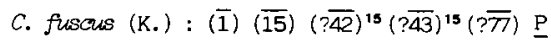

C. Zuriconalis Edw.: ( $\overline{90})$

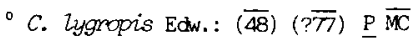

C. ornatus (Mg.) : $(\overline{87})(\overline{90}) \overline{\mathrm{P}} \overline{\mathrm{MC}}$

- C. ascilzator (Mg.) : $(\overline{88})$

- C. pallidipes Edw.: ( $\overline{41})(\overline{48})(\overline{90})$

- C. pimifer Hirv.: ( $\overline{49}) \quad \underline{P}$

* C. pulchripes Verr.: (19) MC

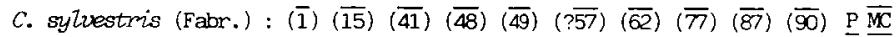

C. tibialis (Mg.) : $(\overline{7})$

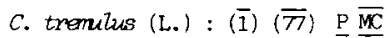

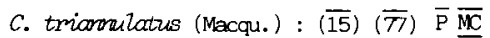

C. tricinctus (Mg.) : $(\overline{48})(\overline{62})(\overline{88})(\overline{90})$ 픔

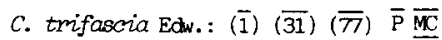

C. trifasciatus (Mg.) : ( $(\overrightarrow{1})(\overline{41})(\overline{48})(\overline{90})$ P $\underline{M C}$

C. viermiensis $\mathrm{G}^{16}:(\overline{31})^{17} \overline{\mathrm{P}} \overline{\mathrm{MC}}$

Diploctadius altiriger $\mathrm{K} .:(\overline{7}) \overline{\mathrm{P}} \overline{\mathrm{MC}}$

* Evkiefferiella alpestris G. : (71) 
E. brevioaloor K.: $(\overline{46})^{18} \overline{\mathrm{P}} \overline{\mathrm{MC}}$

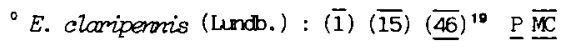

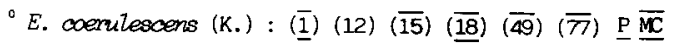

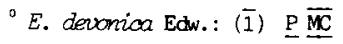

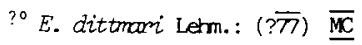

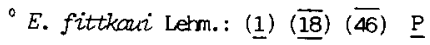

- E. Labifera G.: $(\overline{46})^{20}(\overline{\overline{7}}) \overline{\mathrm{P}} \underline{\overline{\mathrm{MC}}}$

${ }^{\circ}$ E. minar (Edw.) $)^{21}:(\overline{1})(\overline{18})$ (19) (80) $\mathrm{P} \overline{\mathrm{MC}}$

${ }^{\circ}$ E. pseudarortana G. : $(\overline{1})(\overline{18})(\overline{46})^{22} \overline{\mathrm{P}} \mathrm{MC}$

E. tirolensis G.: (므) $(\overline{18})(46)^{23}$ P $\overline{M C}$

* Gmonetriocnonus brumalis (Edw.) : $(\underline{1})(\overline{48}) \underline{P}$

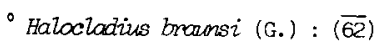

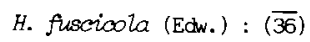

H. stagnomm (G.) ${ }^{24}:(\overline{25})(\overline{30})(\overline{31})(\overline{40})(\overline{90})$

H. varians (Staeg.) ${ }^{26}:(\overline{3})(\overline{87} ; 20(\overline{90})$

${ }^{\circ} H$. vitripermis (Mg.) : $(\overline{55})^{27}$

Heleniella domiem ser.-Tos.: (67) (92)

${ }^{\circ}$ H. ormaticollis (Edw. $)^{20}:(\overline{67})$ P $\overline{\mathrm{MC}}$

-H. serratasioi Ringe : $(\overline{67})^{29}$

Hyotrobaernos distylus K.: (51)

* H. spimatis Saeth.: (토)

Krenasmittia borecalpina G.: (71) $\underline{P}$

Limophyes gragioola Edw.: (18) $\underline{\mathrm{P}} \underline{\overline{\mathrm{MC}}}$

L. pentatomis (K.) : (37)

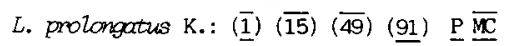

- L. pusillus Eat.: $(\overline{16})(? \overline{82})$

L. smolandiaus $\mathrm{Br} .:(\underline{71}) \underline{\mathrm{P}}$

L. timoni G.: ( $\overline{25})$

Metriocnoms atratulus (Zett.) : (?1)

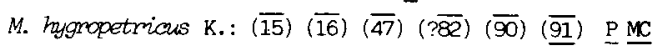

'M. terrester Pag. : ( $\overline{91})$

M. ursinus (Holn.) : (71) $\underline{\mathrm{MC}}$

- Nonoctadius baltious (Pal.) : (49) $\overline{M C}$ 
N. bioolor (Zett.) : ( $\overline{49}) \underline{\mathrm{P}} \underline{\mathrm{MC}}$

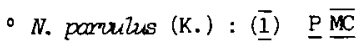

N. rectinerais $(\mathrm{K}):.(\overline{1})(\overline{77}) \overline{\mathrm{P}} \overline{\mathrm{MC}}$

"Orthocladius" bilobatus K.90: (37)

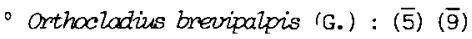

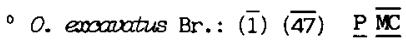

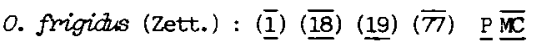

*0 O. fuscimanas (K.) : $(\underline{1})^{31}(\overline{\overline{91}})^{31} \quad \underline{\mathrm{P}} \underline{\overline{\mathrm{MC}}}$

- 0 . fusiformis G. $.^{32}:(\overline{29})(\overline{77})$

O. Lobulifer G.: ( $\overline{31}$ )

O.meridionalis G. : ( $(\overline{31})$

o. mixtus (Holm.) : (71)

o. oblidens $\mathrm{Br} .:(\overline{18})(\overline{49})(\overline{90}) \quad \underline{\mathrm{P}} \underline{\overline{\mathrm{MC}}}$

- O. abtexeens $\mathrm{Br}^{33}:(\overline{1})(\overline{18}) \quad \underline{\mathrm{MC}}$

- O. mivioola K.: ( $(\overline{1})$ (19) $(\overline{49})$ P $\overline{M C}$

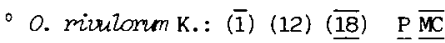

- O. mbicanctis (Mg.) : $(\overline{1})(\overline{6})(\overline{10})(\overline{15})(\overline{17})(\overline{18})(\overline{47})(\overline{48})(\overline{49})(\overline{77})(\overline{83})$ F $\overline{\mathrm{MC}}$

- O. thienemani K.: $(\overline{10}) \quad \underline{\mathrm{P}} \underline{\overrightarrow{\mathrm{MC}}}$

o. timoni G.: $(\overline{31})$

* O. wetterensis $\mathrm{Br} .:(\overline{1})(\overline{77})$

- Paractadius alpicola (Zett.) : $(\overline{49})(\underline{78}) \underline{p}$

P. comersus (Walk.) : $(\overline{49})(\overline{62})(\overline{90}) \overline{\mathrm{P}} \underline{\mathrm{MC}}$

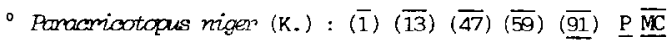

Parakiefferiella gracillima (K.) : (1)

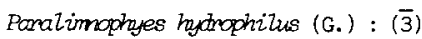

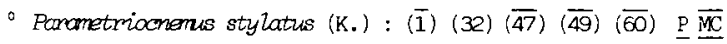

Foraphaenocladius impensus (Walk.) : $(\overline{82})(\overline{91}) \overline{M C}$

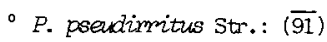

* Paratrichocladius gagi Ser.-Tos.: $(\underline{18})(\underline{\overline{8}})$

- P. hajeralis (G. $)^{34}:(\overline{27})$

P. rufiventris (Mg.) : $(\overline{1})(\overline{15})(\overline{17})(\overline{18})(\overline{47})(\overline{60})(\overline{77})(\overline{83}) \quad \underline{P} \underline{\overline{M C}}$

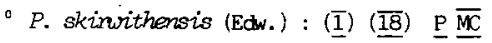

- Paratrissoctadius excerptus (Walk.) : $(\overline{31}) \underline{P} \underline{\overline{M C}}$ 
Pororthocladius radipernis $\left(K_{0}\right)^{35}:(\overline{1})(\overline{6})(\underline{18})(\overline{22})(\underline{23})(\overline{49})(\overline{79}) \underline{P}$

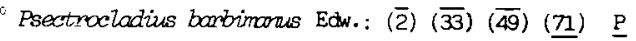

P. bisetus G.: (?94)

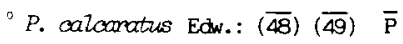

'P. conjingens $(\mathrm{Br}):.(\overline{48})$

P. Linbatellus (Holm. $)^{36}:(\overline{41})(\overline{48})(\underline{71})(7 \overline{82})(\overline{87})(\overline{90}) \quad \overline{\mathrm{P}} \overline{\mathrm{MC}}$

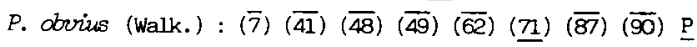

P. sordidellus (Zett.) : $(\overline{30})(\overline{31})(\overline{48})(\overline{49})(\overline{62})(\overline{90}) \quad \underline{P}$

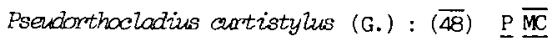

70 P. filiformis (K.) : (?82)

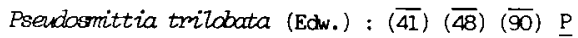

${ }^{\circ}$ Rheocriootopus atripes (K.) ${ }^{37}:(44)(\overline{62})$ P $\overline{M C}$

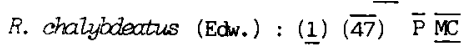

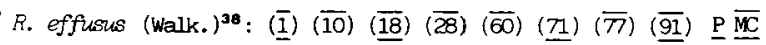

R. fuscipes (K.) : $(\underline{1})(\overline{15})(\overline{44})(\overline{47})(\overline{60})(\overline{77}) \quad \underline{P} \underline{\overline{M C}}$

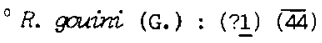

- Smittia alpioola G.: ( $\overline{16})$

S. alpina (K.) : (35)

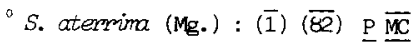

* S. bicolorata (G.) $)^{39}:(\underline{86})$

S. davidi G.: $(\overline{27})$

S. Zongirastris G.: (86)

S. pratorm G. : $(\overline{88}) \overline{(\overline{90})} \overline{\mathrm{P}} \overline{\mathrm{MC}}$

S. thalassicola G.4: ( $\overline{25})(\overline{26})$

Symbiccladius mithrogenae (Zavr.) : (4) $\overline{\mathrm{P}}$

Symorthocladius semivirens (K.) ${ }^{41}:(\overline{1})(\overline{6})(\overline{15})(\overline{17})(\underline{18})(\overline{60})(\overline{77})(\overline{83}) \underline{P} \underline{\overline{M C}}$

* Thienemomia gracei (Edw.) : (1) $\overline{\mathrm{P}} \underline{\mathrm{KC}}$

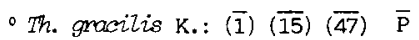

* Th. Liborioa Lav. \& M.: (1)

- Thienencomiella acutioormis $\mathrm{K}^{42}:(\overline{15})(\overline{91}) \overline{\mathrm{P}}$

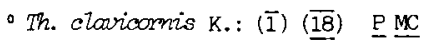

- Th. obscura Br.: $(\overline{15})(\overline{60}) \overline{\mathrm{P}} \overline{\mathrm{MC}}$

Tohomagaia rectangulomis (G.) : (19) (46) ${ }^{43}$ 


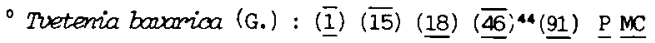

- T. calvescens (Edw. $)^{45}:(\underline{1})(\overline{15})(\overline{18})(\overline{60})(\overline{77})(\underline{91}) \underline{P} \underline{\overline{M C}}$

T. verralli (Edw.) : $(\underline{46})^{4 B}(\overline{49})(\overline{60}) \underline{\mathrm{P}} \underline{\overline{\mathrm{MC}}}$

\section{Girananinae Chiranamini}

Conptochironomus pallidivittatus (Mall.) : $(\overline{41})(\overline{48})(\overline{90}) \mathrm{MC}$

C. tentons (Fabr.) : ( $\overline{41})(\overline{48})(\overline{87})(\overline{90})$

Chironomis amularius (Mg.) : $(\overline{41})(\overline{48})(\overline{49})(\overline{55})(\overline{87})(\overline{90}) \overline{\mathrm{MC}}$

C. conthracinas Zett.: ( $\overline{17})(\overline{49})(\overline{88})(\overline{90})$ P $\overline{\mathrm{MC}}$

C. aprilinas Mg: : $(\overline{41})(\overline{48})(\overline{55})(\overline{87})(\overline{90})$

C. calipterus $\mathrm{K}^{48}:(\overline{40})(\overline{41})(\overline{62})(\overline{87})(\overline{90})$

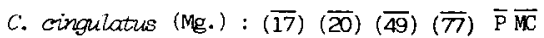

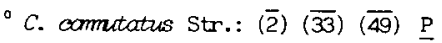

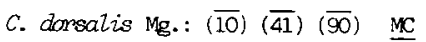

- C. Zongristylus G.: ( $\overline{49})$

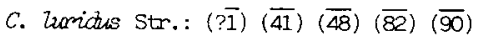

C. Turidus samicinctus Str.: $(\overline{48})(\overline{90})$

C. obtusidens G.: ( $\overline{49})$

C. piger str.: ( $(\overline{41})(\overline{48})(\overline{82})(\overline{87})(\overline{90})$

C. plumosis (L.) : $(\overline{2})(\overline{17})(\overline{33})(\overline{41})(\overline{48})(\overline{49}](\overline{62})(\overline{87})(\overline{90})$ P $\underline{\mathrm{MC}}$

c. psevalothami Str.: (?T) $(\overline{82})(\overline{90})$

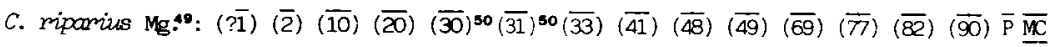

C. salinamius K.: $(\overline{3})(\overline{41})(\overline{48})(\overline{55})$ MC

- C. striatus Str.: (49)

- Cladopelma lateralis (G.) : (51) $\underline{P}$

C. virescens (Mg.): $(\overline{41})(\overline{48})(\overline{90}) \quad \overline{\mathrm{MC}}$

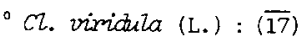

Cryptochironomus rostratus $\mathrm{K}_{.}:(\overline{90}) \overline{\mathrm{P}} \overline{\mathrm{MC}}$

Cr. supplioans (Mg.) : $(\overline{41})(\overline{87})(\overline{90}) \quad \overline{\mathrm{P}} \overline{\mathrm{MC}}$

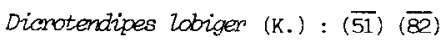

D. modestus (Say; ${ }^{\text {st }}:(\overline{82}) \quad \underline{P}$

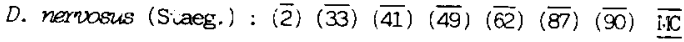

D. notatus (1.g.) : $(\overline{48})(\overline{49})(\overline{82})^{52}(\overline{88})(\overline{90}) \quad \overline{\mathrm{P}} \overline{\mathrm{MC}}$ 


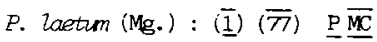

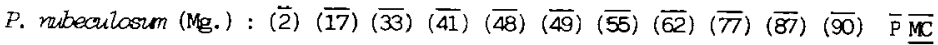

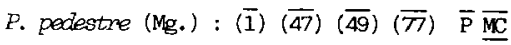

P. phorao K. : $(\overline{40})(\overline{41})(\overline{48})(\overline{62})(\overline{87})(\overline{90})$

P. sondens (v.d.w.) : $(\overline{48}) \overline{\mathrm{P}} \underline{\mathrm{MC}}$

P. tritum (walk.): $(\overline{49})(\overline{82}) \underline{\mathrm{P}} \overline{\mathrm{MC}}$

P. wancinatum (G.) : ( $\overline{82})$

Xenochironam semolabris (K.) : $(\overline{48}) \overline{\mathrm{MC}}$

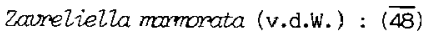

\section{Giranaminee Tarytarsini}

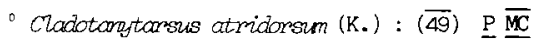

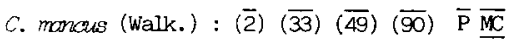

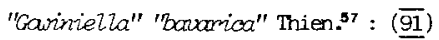

Micropsectra atrofasciata (K.): $(\underline{1})(\overline{18})(\overline{47})(\overline{49})(\overline{60}) \quad \underline{P} \underline{\overline{M C}}$

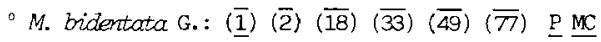

M. clastmieri Reiss : (52)

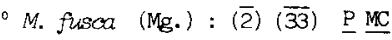

- M. junci (Mg.) : ( $\overline{82}) \quad \underline{\mathrm{PC}}$

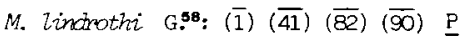

- M. notescenr (Walk.) : ( $\overline{1})$ P $\underline{\overline{M C}}$

o M. praecsc (Mg.) : $(\overline{31})$

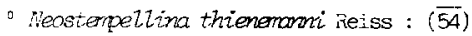

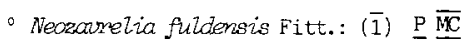

- N. improvisa Fitt.: (13)

Parcopsectra nana (Mg.) : (프)

- Paratanatarsus ausiriacus (K.) : $(\overline{51}) \quad \underline{\mathrm{P}} \overline{\mathrm{MC}}$

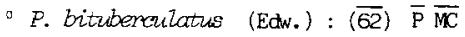

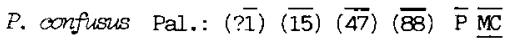

?० P. dimorphis Reiss : (?17)

P. incpertus (Walk.) : $(\overline{77})(\overline{88} ;(\overline{90}) \overline{\mathrm{P}}$

P. lacoophilus (Edw.): (피) $\underline{P}$

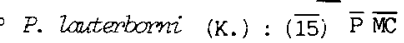


Rheotongtarsus oatistylus (G.) : $(\overline{15})$ P $\overline{M C}$

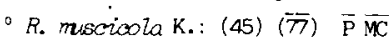

R. nigriocada Fitt.: (1) $\overline{\mathrm{P}} \overline{\mathrm{MC}}$

R. pentacritus $\mathrm{K}^{50}:(39)^{60}$

R. photophitus (G.) : $(\overline{41})(\overline{48})(\overline{87})(\overline{90}) \overline{\mathrm{P}} \overline{\mathrm{MC}}$

R. reissi Letm. : (1) $\overline{\mathrm{KC}}$

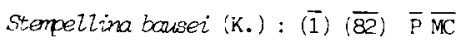

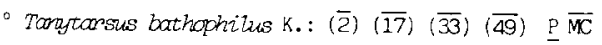

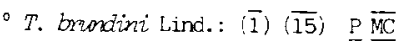

- T. curticomis K. : $(\overrightarrow{49}) \overline{\mathrm{MC}}$

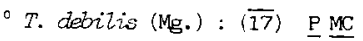

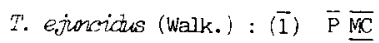

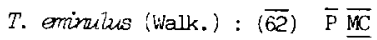

T. fimbriatu Reiss \& Fitt.: $(\overline{49})(\overline{90}) \quad P$

T. gibbasiceps K.: ( $\overline{51}$ )

T. gregarius K.: $(\overline{90})$ MC

T. hausdensis G.: $(\overline{49})(\overline{90}) \quad \underline{P} \underline{\overline{M C}}$

T. holochlorus Edw.: ( $\overline{48}) \mathrm{MC}$

T. homi G." : $(\overline{41})^{62}(\overline{48})(\overline{88})(\overline{90})$

T. Lactescens Edw.: (48)

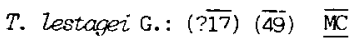

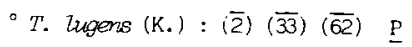

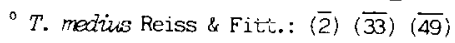

T. Ocuitus Br.: ( $\overline{17})(\overline{90})$

T. palliaicormis (Walk.) ${ }^{03}:(\overline{1})(\overline{49})(\overline{69})(\overline{77})(\overline{82}) \quad \overline{\mathrm{P}} \overline{\mathrm{MC}}$

T. pentomerus (K.) : (39)

T. quadiridentatus $\mathrm{Br} .:(\overline{90})$

T. titia (Tourena) : $(\overline{89})(\overline{90})$

T. usmaensis Pag, : $(\overline{82})(\overline{88})(\overline{90}) \overline{\mathrm{P}}$

T. wolgensis Miseiko : $(\overline{88})(\overline{90})$

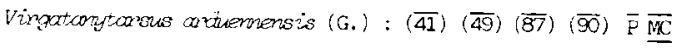

\section{Telmatogetoninae}

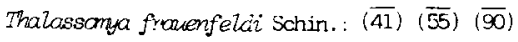


D. peringueyonis K.: ( $\overline{88})$

Einfeldia Longipes (Staeg.) : ( $\overline{82})$

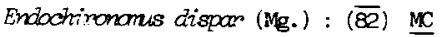

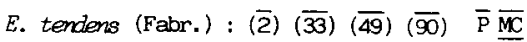

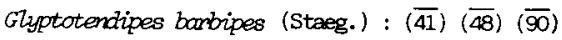

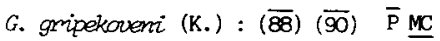

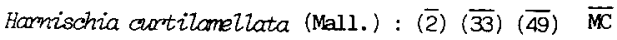

H. fuscimoons $(K):.(\overline{41})(\overline{48})(\overline{49})(\overline{90}) \quad \overline{M C}$

Kiefferulus tendipediformis (G.) : $(\overline{41})(\overline{69})(\overline{82})(\overline{87})(\overline{90}) \quad \overline{\mathrm{MC}}$

Louterborniella agrayloides (K.) : ( $\overline{90})$

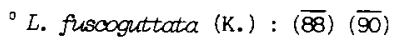

Microchironanus deribae (Frem. $)^{53}:(\overline{3})(\overline{40})(\overline{41})(\overline{87})(\overline{90})$

${ }^{\circ} M$. tener (K.) : ( $(\overrightarrow{48}) \quad \overline{M C}$

Microtendipes diffinis Edw.: $(\overline{30})(\overline{31})$

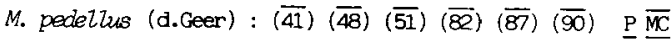

Farachironamus araxtus (G.) : $(\overline{49})(\overline{90}) \overline{\mathrm{MC}}$

P. frequens (Joh. $)^{54}:(\overline{41})(\overline{49})(\overline{87}) \overline{M C}$

P. monochromus (v.d.w.) : ( $\overline{31})$

P. parilis (walk.) : ( $\overline{88})(\overline{90})$

P. vorus (G.) : $(\overline{48})$

P. vitiosus (G.) : $(\overline{49}) \overline{\mathrm{MC}}$

- Paracladopelma mkiona (G.) ${ }^{55}:(\overline{56}) \quad \underline{P}$

Puratauterbormiella nigrohatienalis (wall.) ${ }^{\text {s6 }}:(\overline{90}) \overline{\mathrm{MC}}$

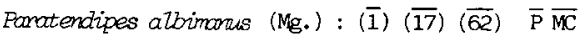

$P$. nudisquama Edw.: (48)

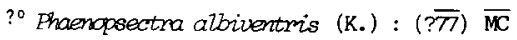

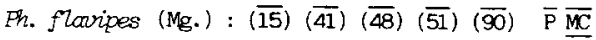

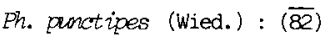

polypedilim acutum $\mathrm{K}_{\mathrm{O}}:(\overline{49}) \overline{\mathrm{P}}$

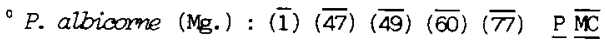

${ }^{\circ}$. barboyoni Ser.-Tos.: ( $\left.\overline{80}\right)(\overline{82})$

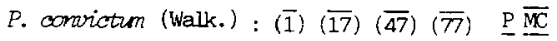

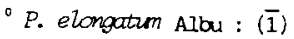


Notes : 1. Selon moi, pas synonyme de Monopelopia tenuicalcar (K.), contrairement à l'opinion de Fittkau (1962). - 2. = Pelopia tetrasema K. - 3. = punctulata G. ; = Heptagia sp. A Saunders (1930) - 4. = Heptagia sp. B Saunders (1930). - 5. = berardensis Ser. -Tos. - 6. Sub 0. berardensis n. sp. et sub D. latitarsis 6. - 7. Larves, identification douteuse. - 8. Localisation en France non précisée. - 9. Larves, identification douteuse. - 10. = thienemanni $K$. 11. Larves, identification douteuse. - 12. = Syndiamesa pubitarsis auct. - 13. Sub Diamesa hygropetrica K. - 14. = lucidus Staeg. - 15. Sub Cricotopus biformis Edw. - 16. = pseudosinilis G. E T.D. - 17. Sub C. pseudosimilis n. $\$$. et sub C. decorus G. - 18. Les spécinens de E. brevicalcar cités de France par Lehmann (1972) proviennent des Alpes (Vaulnaveys-le-Bas, Isère, $310 \mathrm{~m}, 1.3 .1964$, Serra-tosio leg.; Goncelin, Isère, $235 \mathrm{~m}, 18.10 .1956$, Vaillant leg.) et du Massif Central. - 19. Les spécimens de E. clarioennis cités de France par Lehmann (1972) proviennent des Alpes (massif du Vercors, Engins, $965 \mathrm{~m}, 19.4 .1964$. Serra-Tosio leg. ; Villardde-Lans, 980 n, 7.3.1966, Serra-Tosio leg. ; massif de Chartreuse, le Sappey, 1010 m, nars 1963, Serra-Tosio leg.) et du Massif Central. - 20. Les spécimens de E. lobifera cités de France par Lehmann (1972) ont été capturés dans les Alpes (Grenoble, $220 \mathrm{~m}, 22.4 .1964$, SerraTosio leg. ; Goncelin. Isère, $235 \mathrm{~m}, 18$ et 28.10 .1966 , Vaillant leg.) et le Massif Central. 21. - Eukiefferiella mirabilis Ser.-Tos., nov. syn. : j'ai examiné un nombre élevé de spécimens de E. minor (Edw.) capturés dans les Alpes depuis quelques années. Leurs caractères s'accordent en général assez bien avec la description que donne Lehanan (1972) de cette espèce, sauf en ce qui concerne le gonostyle. Ce dernier est souvent comme le décrit Lehmann, mais parfois la sorte de cassure ("Knick") signalée comme caractéristique de E. minor par cet auteur manque to talement, comme chez E. mirabilis. De plus, lors du montage en préparation microscopique, le gonostyle de certains spécimens peut se retourner de $180^{\circ}$, prenant alors l'allure étrange que je croyais typique de E. mirabilis. Par ailleurs, les autres caractères de l'imago mâle de E. mirabilis ne différant pas significativement de ceux de E. minor. je considère que Eukiefferiella mirabilis Serra-Tosio, 1983 est un symonyme junior de Eukiefferiella minor (Edw.). 22. Les spécimens de E. pseudomontana cités de France par Lehmann (1972) ont été capturés dans les Alpes (Noyarey, lsère, $569 \mathrm{~m}, 22.3 .1966$, Serra-iosio leg.) et dans le Massif Central. 23. Les spécimens de E. tirolensis cités de France par Lehmann (1972) proviennent des Alpes (Vanoise, Savoie, Plan du Lac, 2355 n, 10.7.1968, Serra-Iosio leg.). - 24. = maritimus G. 25. = halophilus K. - 26. Sub Cricotopus vitripennis var. halophilus K. - 27. L'identification de cet insecte est peu sûre. - 28. = intermedia Ser.-Tos. - 29. Sub ornaticollis Edw. - 30 . Appartenance au genre Orthocladius pas sûre (Fittkau f, Reiss, 1978). - 31. Sub bipunctellus Zett. - 32. Selon moi, probablement synonyme de rivicola K. - 33. Selon Cranston (1984), peutêtre synonyme de fuscimanus K. - 34. Selon Hirvenoja (1973), peut-être synonyme de rufiventris Mg. - 35. = curtistylus G. ; = curtistylatus G. ; = torrentium G. - 36. = edwardsi Br. -37. = foveatus EdH.-38. = dorieri $\overline{G .-39 . ~ S e l}$ on Hirvenoja (1973), appartenance au genre Snittia seulement probable. $-\mathbf{4 0}=$ thalassobia G. - 41. = tripilatus Edw. - 42. = fusca K. - 43. Les spécimens de cette espèce cités de France par Lehmann (1972) ont été capturés dans les Alpes (col du Lautaret, Hautes-Alpes, $2040 \mathrm{~m}, 17.7 .1963$, Serra-Tosio leg.). - 44. Les spécimens de cette espèce cités de France par lehmann (1972) proviennent des Alpes (massif de la Chartreuse, le Sappey, 1010 m, mai 1963, Serra-Tosio leg. ; nassif de Belledonne, le Mas Jullien, $753 \mathrm{~m}, 28.4 .1963$, Serra-Tosio leg. ; massif du Vercors, Montaud, $780 \mathrm{~m}, 22.3 .1966$, Serra-Tosio leg.l. - 45. Les spécimens de cette espèce cités par erreur de France par Lehmann (1972) proviennent en fait de Suisse (Grisons, le Poschiavino à Miralago, 14.8.1961, Serra-Josio leg.). 46. Les spécimens de cette espèce cités de France par Lehmann (1972) proviennent des Alpes (massif du Vercors, Villard-de-Lans, $980 \mathrm{~m}, 7.3,1966$, Serra-Tosio leg.). - 47. = halophilus $\mathrm{K}$. 48. = hexasticus K. - 49. = thumi K. - 50. Sub dorsalis var. riparius $-51 .=\underline{\text { pulsus }}$ (Walk.). - 52. Y compris 0. notatus notatus (Mg.) et D. notatus rufolineatus (G.). -

53. = paraderibae Lav. E Tour. - 54. = longiforceps K. - 55. = schlitzensis Ringe. - 56. = brachylabis Edw. - 57. Selon Reiss (1969), ce nom de genre et ce non d'espèce ne sont pas valides (nomen nudum), car le manuscrit dans lequel Thienemann devait les créer n'a jamais fait l'objet d'une publication. D'après Reiss (op. cit.), il pourrait s'agir d'un Chironomidé du genre Micropsectra. - 58. = foliata Lav. - 59. Considéré par Lehmann (1970) comme nomen dubiup. 60. Cité par Kieffer $\overline{(1922)}$ du Dauphiné sans autres précisions géographiques. - 61. = nigrocinctus Fr. - 62. Sub I. gr. holochlorus Edw. - 63. = paschalis 6 . 
Mais à mon avis, cela est difficile et prématuré : les Chironomidés des Alpes françaises sont vraiment trop mal connus. Ainsi, un nombre élevé d'espèces trouvées dans les Pyrénées au-dessus de $1000 \mathrm{~m}$ d'altitude et dans le Massif Central au-dessus de $500 \mathrm{~m}$ n'ont pas, jusqu'à présent, été signalées des Alpes françaises au-dessus de $1000 \mathrm{~m}$, mais sont présentes dans les vallées alpines à des altitudes parfois très inférieures (jusqu'à moins de $200 \mathrm{~m}$ ). A cet égard, le cas extrême des Chironomini, dont une seule espèce a été mentionnée des Alpes au-dessus de $1000 \mathrm{~m}$, est particulièrement éloquent.

L'état du peuplement en Diptères Chironomidae des Alpes et des basses régions avoisinantes qui est donné ici constitue donc plus une incitation à poursuivre et à compléter les recherches antérieures qu'une base solide et étoffée pour des travaux comparatifs satisfaisants de biogéographie.

Enfin, quand on fait le décompte de tous les Chironomidés trouvés dans les régions situées au sud de la Loire et du Rhône français en amont de Lyon, soit à peu près la moitié sud de la France, on at teint le chiffre de 496 espèces. Il se décompose en 49 Tanypodinae $(9,9 \%)$, I Buchonomyinae $(0,2 \%), 36$ Diamesinae $(7,3 \%), 5$ Prodiamesinae $(1,0 \%), 215$ Orthocladiinae $(43,3 \%), 189$ Chironominae $(38,1 \%)$ dont 107 Chironomini $(21,6 \%)$ et 82 Tanytarsini $(16,5 \%)$, et 1 Telmatogetoninae $(0,2 \%)$.

Ce total des Chironomidés connus dans la seule moitié sud de la France, qui approche donc les 500 espèces, est supérieur aux chiffres indiqués par Laville \& Serra-Tosio (1987) pour les Chironomidés de l'Irlande ( 347 espèces), de la Roumanie ( 407 espè. ces) ou de la Grande-Bretagne (448 espèces). Le total des Chironomidés connus de la France entière, dont le catalogue reste à faire, est donc encore plus élevé. On peut par conséquent considérer la faune française de ces Diptères comme relativement riche et variée, comparée aux quelques 1300 espèces de Chironomidés répertoriêes dans la totalité de la région paléarctique (Ashe, Murray \& Reiss 1987).

\section{Travaux cités}

- Ashe (P.), Murray (D.A.) \& Reiss (F.). 1987. - The zoogeographical distribution of Chironomidae (Insecta : Diptera). Annls Limnol. 23 : 27-60.

1. Buisson (J.). 1986. - Hydrobiologie du massif du Vercors (Préalpes calcaires) et d'une rivière-type : le Furon. Ecologie des Dipteres Chironomidae du Furon et de quelques cours d'eau pollués. Thèse de Doctorat de 3 e cycle, Université Scientifique, Technologique et Médicale de Grenoble : $191 \mathrm{p}$.
2. - Champeaux (A.) et al. 1982. - Les retenues hydroélectriques du Verdon : impact sur la rivière, conséquence du marnage. Bull. Ecol., 13 (2) : 203-239.

3. - Cheraitia (M.L.). 1984 - Essai d'appréciation de la qualité de quelques milieux stagnants de faible profondeur à partir des macroinvertébrés. Application aux bassins d'épuration par lagunage. Thèse de Doctorat de 3 eycle, Univ. ClaudeBernard, Lyon I: $99 \mathrm{p}$.

4. - Codreanu (R.). 1939. - Recherches biologiques sur un Chironomide, Symbiacladius thithrogenae (Zavr.), ectoparasite " cancérigène * des Ephémères torrenticoles. Arch. Zool. Exp. Gen., $81 ; 1.283$.

5. - Dorier (A.). 1926. - Un Chironomide (Dactylocladius brevipalpis Goetghebuer) a larve commensale d'une nymphe d'Ephéméride (Rhithrogena semicolorata Curt.). Trav. Lab. Hydrobiol. Grenoble, 18 : 63-73.

6. - Dorier (A.) 1933 a. - Les métamorphoses de quelques Orthocladiinae recueillis en Dauphiné. Trav. Lab. Hydrobiol. Gre. noble, $25: 191.202$.

7. - Dorier (A.). 1933 b. - Sur la biologie et les métamorphoses de Psectrocladius obvitus Walk. Trav, Lab. Hydrobiol. Grenoble, 25 : 205-215.

8. Dorier (A.), 1937. - La faune des eaux courantes alpines. Verh. irtt. Vereinig. Limnol., 8 (1): 33-41.

9. - Dhorier (A). 1938. - Une nouvelle station de Dactylocladius brevipalpis Goet., Chironomide parasite de nymphes d'Ephémères. Bull. Soc Entomol. Fr. 43 : 45.46.

10. - Dorier (A.). 1939 a. - La répartition des larves de Chironomides dans les eaux courantes polluées par des matières organiques. C.R. Ass. Fr. Av. Sc.: 649-653.

11. - Dorier (A). 1939 b. - Notes faunistiques sur quelques lacs du massif de Chambeyron (Basses-Alpes). Trav. Lab. Hydrobiol. Grenoble, 27-29:25-30.

12. - Dorier (A.) \& Vaillant (F.). 1954. - Observations et expérien. ces relatives à la résistance au cou rant de divers Invertébrés aquatiques. Trav. Lab. Hydrobial. Grenoble, 45-46:9-31.

13. - Fittkau (I.J.). 1954. - Die Gattung Neozavrelia Goetghebuer (Dipt. Chironomidae). Chironomidenstudien II. Deuts. ent. Z, $1(3-5): 161+179$.

14. - Fittkau (E.J.). 1962, - Die Tanypodinae (Diptera : Chironomidae). Die Tribus Anatopinyini, Macropelopiini und Pentaneurini. Abh. Larvalsystemat. Insekten, 6; 453 p.

15. - Flueler (M.J.). 1986. - Etude des Macroinvertébrés - surtout des Diptères - pour trois ruisseaux de plaine. Thèse de $3^{\text {e }}$ cycle, Université Scientifique et Médicale de Grenoble: $128 \mathrm{p}$.

16. - Gachet (M.), 1970. - Etude des fluctuations de la faune ripicole d'un étang astatique des environs de Grenoble. Thèse de $3^{e}$ cycle, Univ. Grenoble, vol. I : 62 p.; vol. II : 86 p.

17. - Gay (Ch.). 1976. - Contribution à l'étude écologique et à l'aménagement d'un lac de montagne: le lac de Petichet (Isère). Thèse de $3=$ cycle, Univ. Grenoble : $124 \mathrm{p}$.

18. - Gay (Ch). 1982 a. - Les communautés benthiques d'un torrent des Alpes françaises: l'Eau d'Olle (Isère). Trav. Lab. Hydrobiol. Grenoble, 71-73: 7-31

19. - Gay (Ch.). 1982 b. - La faune benthique d'un torrent glaciaire des Alpes françaises : la Romanche au Plan de l'Alpe (HautesAlpes). Trav. Lab. Hydrobiot. Grenoble, 71-73:33-44.

20. - Gay (Ch.) \& Serra-Tosio (B.). 1982. - L'influence de barrages sur l'écologie d'une rivière polluée : la basse Isère en aval de Grenoble. Trav. Lab. Hydrobiol. Grenoble, $71-73$ : 45-58.

21. - Goetghebuer (M.). [928. - Description d'un Chironomide du groupe Diamesa (Syndiamesa nivosa n. sp.). Diptera, 4: 123-128,

22. - Goetghebuer (M.). 1933 a - Ceratopogonidae et Chironomjdae nouveaux ou peu connus d'Europe (troisième note). Bull. Ann Soc ent. Belg. $73: 209-221$. 
23. - Goetghebuer (M.). 1933 b. - Ceratopogonidae et Chironomidae nouveaux ou peu connus d'Europe. $4^{*}$ note. Bull. Ann. Soc ent. Belg., 73: 353-361.

24. - Goetghebuer (M.). 1936. - Tendipedidae (Chironomidae) a) Subfamilie Pelopiinae (Tanypodinae) A. Die Imagines. $\boldsymbol{l} \boldsymbol{n}: \mathbf{E}$. Lindner, die Fliegen der Paläarktischen Region, 13 b : $1-50$.

25. - Gotghebuer (M.) 1937. - Ceratopogonidae et Chironomidae nouveaux ou peu connus d'Europe (septième note). Bull. Ann. Soc. ent. Belg., $77: 273-280$.

26. - Goetghebuer (M.). 1940-1950. - Tendipedidae (Chironomidae) f) Subfamilie Orthocladiinae. A. Die Imagines. I $\mathrm{t}$ : E. Lindner, die Fliegen der paläarktischen Region, $13 \mathrm{~g}$ : 1-208.

27. - Goetghebuer (M.). 1950. - Ceratopogonidae et Chironomidae nouveaux ou peu connus d'Europe (14e note). Bull. Inst. Sci. nat. Belg. $26(47): 15 \mathrm{p}$.

28. - Goetghebuer (M.) \& Dorier (A.). 1932. - Les métamorphoses d'Orihocladius (Dactylocladius) dorieri Goetghebuer. Bull. Lab. Hudrobiol. Grenoble, 23 : 61-65.

29. - Goetghebuer (M.) \& Dorier (A.). 1939. - Description sommaire de la nymphe et l'adulte d'Orthocladius fusiformis Goetgh. Bull. Soc. ent. Fr., $44:$ 30-32.

30. - Goetghebuer (M.) \& Timon-David (J.). 1937. - Contribution à l'étude des Diptères halophiles et halobies du littoral méditerranéen. Chironomides et Cératopogonides de l'îlot de Planier. Bull. Ann. Soc. ent. Belg., 77 : 409.416

31. Goetghebuer (M.) \& Timon-David (J.). 1939. - Nouvelles observations sur les Chironomides et Cératopogonides marins des îles du golfe de Marseille. Bull. Ann. Soc ent. Belg., $79: 63-70$.

32. - Gouin (F.) 1956. Parametriocnemus stylatus K. et P. arciger K. (Dipt. Némat. Chironomidae) : deux espèces ou deux variétés ? Bull. Ass. philomath. Alsace-Lorraine, 9 (4) : 194-198.

33. - Grégoire (A.). 1982. - Contribution à l'étude hydrobiologique d'une rivière aménagé : le Verdor. Les lacs de barrage et les tronçons de cours d'eau à débit régulé. Cahiers Lab. Hydrobiol. Montereau, $13: 172 \mathrm{p}$.

34. - Hansen (D.C.) \& Cook (E.F.). 1976. - The systematics and morphology of the nearctic species of Diamesa Meigen, 1835 (Diptera, Chironomidae). Mem. Amer. Ent. Soc., 30:1-203.

35. - Hubault (E.). 1927. - Contribution à l'étude des Invertébrés torrenticoles. Bull. biol. Fr. Belg., suppl, 9: 1-389.

- Illies (J.). 1967. - Limnofauna Europaea. G. Fischer Verlag, Stuttgart : $\mathbf{4 7 4} \mathrm{p}$.

- Illies (J.). 1978. - Limnofauna Europaea. G. Fischer Verlag, Stuttgart, New York ; Swets \& Zeitlinger B.V., Amsterdam : $532 \mathrm{p}$.

36. - Jolivet (P.). 1973. - Le problème des Chironomides (Diptères Nématocères) dans le Languedoc-Roussillon (note préljminaire). Vie et Milieu, 23 (2 C) : 269-290.

37. - Kieffer (J.J.). 1915. - Neue Chironomiden aus Mitteleuropa. Broteria Braga, $13: 65-87$.

38. - Kieffer (J.J.). 1921. - Neue Chironomiden aus Mitteleuropa. Arch. Hydrobiol., Suppl. 2: 785-808.

39. - Kieffer (J.J.). 1922. - Chironomides nouveaux ou peu connus de la région paléarctique. Ann. Soc. sci. Bruxelles, 42 : 71-128 et 138-180.

- Laville (H.). 1980. - Inventaire 1980 des Chironomides (Diptera) connus des Pyrénées. Annis. Limnol., 16: 211-223.

- Laville (H.) \& Serra-Tosio (B.). 1987, - Chironomidés (Dip tera) du Massif Central et des basses régions avoisinantes. Annls Limnol., 23 : 135-145.

40. - Laville (H.) \& Toureng (J.N.). 1967, - Contribution a la connaissance de trois Chironomides de Carnargue et des Marismas du Guadalquivir (Diptères). Annls Limnol, 3 ; 185.204,

41. - Laville (H.) \& Toureng (J.N.), 1968, - Nouvelles récoltes de Chironomides en Camargue et dans les Marismas du Guadalquivir. Annls Limnol., 4 : 73-80.
- Laville (H.) \& Vinçon (G.). 1986. - Inventaire 1986 des Chjromidés (Diptera) conruss des Pyrénées. Annls Limnol., 22 : 239-251.

42. - Léger (L.) \& Motas (C.) 1928 a - Biologie d'un Chironomide, le Cricotopus biformis et son intérét en salmoniculture. Trav. Lab. Hydrobiol. Grenoble, $20: 1-18$.

43. - Léger (L.) \& Motas (C.) 1928 b. - Parasitisme et phénomène de transport d'un Hydracarien chez un Chironomide du genre Cricotopus. C.R. Acad. Sci. Paris : 1238-1239.

44. - Lehmann (J.), 1969. - Die europäischen Arten der Gattung Rheocricotopus Thien. und Harn. und drei neue Artvertreter dieser Gattung aus der Orientalis (Diptera, Chironomidae). Arch. Hydrobiol., 66 (3) : 348-381.

45. - Lehamnn (J.). 1970. - Revision der europäischen Arten (Imagines $\sigma \sigma$ und Puppen $\odot$ Q) der Gattung Rheotanytarsus Bause (Diptera, Chironomidae). Zool. Anz, 185:345-378.

46. - Lehmann (J.). 1972. - Revision der europäischen Arten (Puppen $\sigma o$ und Imagines $Q$ ९) der Gattung Eukiefferiella Thienemann (Diptera: Chironomidae), Beitr. Ent, 22 (7/8): $347-405$.

47. - Madariaga-Meza (M.). 1984 - Un ruisseau de basse montagne, le Sonnant (Isère). Etude de l'influence d'une station d'épuration. Thèse de $3^{e}$ cycle, Univ. Scientif. et Méd. de Grenoble : $64 \mathrm{p}$.

48. - Moubayed (Z.). 1978. - Etude écologique des marais de la Crau (B. du Rh.). Analyse des peuplements d'Invertébrés dulçaquicoles et leurs relations avec l'hydrologie, la végétation et les influences humaines. Thèse de $3^{\text {e }}$ cycle, Univ. AixMarseille III : $222 \mathrm{P}$

49. - Nino (A.). 1982. - La faune benthique du lac de barrage de Serre-Ponçon. Structure du peuplement, ecologie des Chironomides. Thèse de $3^{2}$ cycle, Univ. de Provence Aix-Marseille I : $196 \mathrm{p}$

50. - Pagast (F.) 1947. - Systematik und Verbreitung der um die Gattung Diamesa gruppierten Chironomiden. Arch. Hydrobiol., 41 : 435-596.

51. - Reiss (F.). 1968. - Verbreitung lakustrischer Chironomiden (Diptera) des Alpengebietes. Ann. Zool. Fenn., 5 : 119-125.

52. - Reiss (F.) 1969 a. - Revision der Gattung Micropsectra Kieff., 1909 (Diptera, Chironomidae). 1. Die attenuata Gruppe der Gattung Micropsectra. Beschreibung 5 neuer Arten aus Mitteleuropa und Nordafrika. Dtsch. Ent. Z., N.F. $16(4 / 5)$ : 431-449.

53. - Reiss (F.). 1969 b. - Die neue, europäisch verbreitete Chironomidengattung Parapsectra mit einem brachypteren Artvertreter aus Mooren (Diptera). Arch. Hydrobiol., 66 (2) : 192-211.

54. - Reiss (F.). 1984. - Neostempellina thienemanni n. gen. n. sp., eine europäische Chironomide mit gehäusetragenden Larven (Diptera, Insecta). Spixiana, 7 (2) : 203-210.

55. - Remmert (H.). 1953. - Les Diptères des côtes méditerranéennes de France et d'Espagne. Vie et Milieu, 4 (3) : 540-546.

56. - Ringe (F.). 1974. - Chironomiden.Emergenz 1970 in Breiterbach und Rohrwiesenbach. Schlitzer Produktionsbiologische Studien X. Arch. Hydrobiol., Suppl. 5 (2/3) : 212-304

57. - Risbec (J.). 1951. - Les Diptères nuisibles au riz de Camargue au début de son développement. Rev. Palh. vég. Ent.agr. Paris, $30: 211-227$.

58. - Saether (O.A.) 1976. - Revision of Hydrobaenus, Trissocladius, Zalutschia, Paratrissocladius, and some related genera (Diptera, Chironomidae). Bull. Fish. Res. Board Can., 195 : $287 \mathrm{p}$.

59. - Saether (O.A.). 1980. - The females and immatures of Paracricotopus Thienemann and Hamisch, 1932, with the description of a new species (Diptera, Chironomidae). Aquatic Insects, 2 (3) : 129.145

60. - Salman (S.A.). 1979. - Etude écologique d'un ruisseau de plaine * le Fontanil $•$ Thèse de $3 *$ cycle, Univ. Grenoble : $72 \mathrm{p}$. 
61. - Saunders (L.G.). 1930. - The larvae of the genus Hepragyia with description of a new species. Ent. Mon. Mag., 66 : 209.214.

62. - Séguier (J.). 1981. - Etude des relations entre les écosystèmes de sources, de ruisseaux et de marais dans un complexe limnique du nord de la $C_{\text {rau. }}$ Thèse de 3 cycle, Univ. AixMarseille (Saint-Jérôme) : $246 \mathrm{p}$.

63. - Serra-Tosio (B.) 1964 a. - Quelques Dianesini (Diptera Chironomidae) du Dauphiné et du Vivarais. Description de quatre especes nouvelles. Traw Lab. Hydrobiol. Grenoble, 56: 29-52

64. - Serra-Tosio (B.). I964 b. - Prodiamesa (Monudiamesa) delphinensis $\mathrm{n}$. sp., une nouvelle espèce de Chironomidae (Dip tera) de la région grenobloise. Trav. Lab. Hydrobiol. Greno ble, $56: 97 \cdot 103$

65. - Serra-Tosio (B.) 1966. - Nouveltes données concernant la répartition et l'écologie de quelques Chironomides Diamesini dans le sud-est de la France. Gewässer und Abwässer, 41/42: 124-128.

66. - Serra-Tosio (B.) 1967 a - Taxonomie et écologie des Diamesa du groupe latitarsis (Diptera, Chironomidae). Trav. Lah. Hydrobiol. Grenoble, 57-58:65-91.

67. - Serra-Tosio (B.). 1967 b. - Sur les Orthocladiinae du genre Heleniella Gowin (Diptera, Chironomidne), Dtsch. Ent. Z., N.F. $14(1 / 2): 153-162$

68. - Serra-Tosio (B.). 1968. - Taxonomie phylogénétique des Diamesini : les genres Potthastia Kieffer, Sympotthastia Pagast Parapotthastita n.g, et Lappodiamesa n.g. (Diptera, Chirono midae). Trav. Lab. Hydrobiol. Grenoble, 59-60: 117-164.

69. - Serra-Tosio (B.). 1970 a. - Quelques aspects écologiques du peuplement d'une petite mare. Trav. Lab. Hydrobiol. Greno ble, 61 : 33-67.

70. - Serra Tosio (B.) 1970 b. - Les Diamesa du groupe dampfi. Description d'une espece nouvelle (Diptera, Chironomidae) Trav. Lab. Hydrobiol. Grenoble, 61: 107-146.

71. - Serra-Tosio (B.). 1970 c. - Quelques Dipteres Chironomides, Simuliides et Culicides du parc national de la Vanoise. Trav. Scient. Parc Nat. Vanoise, $1: 119-129$.

72. - Serra-Tosio (B.), 1971. - Contribution à l'étude taxonomique phylogénétique, biogéographique et écologique des Diamesini (Diplera, Chironomidae) d’Europe, Thèse Doct. d'Etat, Univ. Grenoble, 2 volumes : $462 \mathrm{p}$.

73. - Serra-Tosio(B.) 1972. - Description et écologie de Diamesa vaillanti n. sp. (Diptera, Chironomidae). Trav. Scient. Parc Nat. Vanoise, 2 : 9.25 .

74. - Serra-Tosio (B.) 1973 (daté 1972). - Ecologie et biogéographie des Diamesini d'Europe (Diptera, Chironomidae). Trav. Lab. Hydrobiol. Grenoble, $63: 5-175$

75. - Serra-Tosio (B.). 1974. - La mouche des glaciers Diamesa steinboecki Goetgh., insecte de montagne à atles réduites (Diptera, Chironomidae). Trav. Scient. Parc Nat. Vanoise, 5 : $165-189$

76. - Serra-Tosio (B.). 1976. - Chironomides des Alpes : le genre Psetudodiamesa (Diptera, Chironomidae). Trav. Scient. Parc Nat. Vanoise, $7: 117-138$

77. - Serra-Tosio (B.). 1977. - Note sur les Diptères Chironomides de quelques rivières polluées dans la région de Grenoble. Tra1: Lab. Hydrobiol. Grenoble, 66-68: 83-88.
78. - Serra-Tosio (B.). 1978. - Les Diptères Chironomidés du lac du Mont-Coua (Parc National de la Vanoise). Trav. Scient. Parc Nat. Vanoise, $9: 141-145$

79. - Serra-Tosio (B.). 1981. - Contribution à l'étude du genre Parorthocladius Thien. (Dipt. Chironornidae). Bull. Soc, ent. Fr., $86: 217-223$.

80. - Serra-Tosio (B.). 1983. - Trois Chironomidés nouveaux de la Région Rhóne-Al pes (Dipt. Nematocera). Bull. Soc. ent. Fr. $88: 12-20$.

81. - Serta-Tosio (B.) 1989. - Révision des espèces onest. paléarctiques et néarctiques de Boreoheptagyia (Diptera, Chironomidae), avec des clés pour les larves, les nymphes et les imagos. Spixiana, 11 (2) : 133-173.

82. Serra+Tosio (B.) \& Barboyon (P.) 1981 - Les Chironomidae (Diptera) des milieux peu profonds de la vallée du Rhône en amont de Lyon. In : Les milieux aquatiọues de lisière dans la vallée du Rhóne en amont de Lyon, tome Il. Ministère de I'Environnement. Laboratoires de Zoologie et de Botanique, Univ. Grenoble : $107 \mathrm{p}$.

83. - Serra-Tosio (B.) \& Gay (Ch.). 1978. - Les Diptères Chirono midés et Chaoboridés du lac de Pet ichet (Isère). Trav. Lab. Hydrobiol. Grentoble, 69-70:97-105.

84. - Thienemann (A.). 1952 a. - Diamesa parva. Zool. Anz., 149 : 40-42.

85. - Thienemann (A), 1952 b. - Bestimmungstabelle für die Larven der mit Diamesa nachst verwandten Chironomiden. BetIräge zur Entomologie, 2 : 244-256.

86. - Timon-David (J.). 1937. - Recherches sur le peuplement des hautes montagnes. Diptères de la vallé de Chanonix et du massif du Mont-Blanc. Ann. Fac. Sci. Marseille, $10: 5-54$.

87. - Tourenq (J.N.). 1966. - Introduction à l'étude écologique des Chironomides des eaux douces et saumâtres de Camargue (Diptères). Annls Limnol., 2 ; 459-465.

88. - Tourenq (J.N.). 1970. - Complément à l'inventaire des Chironomides de Camargue (Dipt.). Annls Limnol., 6 ; 363-370.

89. Tourenq (J.N.). 1975. - Recherches écologiques sur les Chironomides (Diptera) de Camargue. Thèse Doct. d'Etat, Univ. Toulouse : $424 \mathrm{p}$

90. - Tourenq (J.N.). 1976. - Recherches écologiques sur les Chironornides (Diptera) de Canargue. I. Etude faunistique et biogéographique. Annls Limnol., $12: 17.74$.

91. - Vaillant (F.). 1955. - Recherches sur la faune madicole de France, de Corse et d'Afrique du Nord. Mém. Mus. Hist. nat. Paris (A) 11 : 1-258.

92. - Vaillant (F.), Degrange (C.) \& Serra-Tosio (B.). 1972. - Ia faune de l'Isère et de quelques-uns de ses tributaires. Trav. Lab. Hydrobiol. Grenoble, 63:189.213.

93. - Verneaux (J.) \& Vergon (J.P.)- 1974. - Faune dulçaquicole de Franche-Comté. Sixième partie : les Diptères Chironomi dés. Ann Scient. Univ. Besançon (Zool., Physiol. et Biol. Anim.), 3e série, $11:$ 179-198.

- Wasson (J.G.). 1975. - Etude écologique d'une rivière pol luée : l'Isère à l'aval de l'agglomération grenobloise. Thèse de $3^{e}$ cycle, Univ. Grenoble: $116 \mathrm{p}$.

94. - Wülker (G.). 1956. - Zur Kenntnis der Gattung Psectrocla dius Kieff. (Dipt. Chironomidae). Individuelle Variabilität. Grenzen und Möglichkeiten der Artent rennung, Okologie und Verbreitung. Arch. Hydrobiol., Suppl. 24 (1) : 1-66. 